\title{
Estimations of the second coefficient of a univalent, bounded, symmetric and non-vanishing function by means of Loewner's parametric method
}

\author{
by J. ŚlLadKOWska (Gliwice)
}

\begin{abstract}
Let $\mathcal{B}_{0}^{(R)}(b)$ denote the class of functions $F(z)=b+A_{1} z+A_{2} z^{2}+\ldots$ analytic and univalent in the unit disk $U$ which satisfy the conditions: $F(U) \subset U, 0 \notin$ $F(U), \operatorname{Im} F^{(n)}(0)=0$. Using Loewner's parametric method we obtain lower and upper bounds of $A_{2}$ in $\mathcal{B}_{0}^{(R)}(b)$ and functions for which these bounds are realized. The class $\mathcal{B}_{0}^{(R)}(b)$, introduced in [6], is a subclass of the class $\mathcal{B}_{u}$ of bounded, non-vanishing univalent functions in the unit disk. This last class and closely related ones have been studied by various authors in [1]-[4]. We mention in particular the paper of D. V. Prokhorov and J. Szynal [5], where a sharp upper bound for the second coefficient in $\mathcal{B}_{u}$ is given.
\end{abstract}

1. Introduction. Let $\mathcal{B}_{0}^{(R)}(b), 0<b<1$, denote the class of all functions $F$ that are analytic, univalent in the unit disk $U$ and satisfy the conditions

$F(U) \subset U, F(0)=b, 0 \notin F(U), \operatorname{Im} F^{(n)}(0)=0, n=0,1, \ldots, \quad F^{\prime}(0)>0$.

Let

$$
F(z)=b+A_{1} z+A_{2} z^{2}+\ldots, \quad A_{1}>0
$$

and

$$
L(z)=K^{-1}\left(\frac{4 b}{(1-b)^{2}}\left(K(z)+\frac{1}{4}\right)\right)=b+B_{1} z+B_{2} z^{2}+\ldots,
$$

where $K(z)=z /(1-z)^{2}$,

$$
B_{1}=\frac{4 b(1-b)}{1+b}, \quad B_{2}=\frac{-8 b(1-b)\left(b^{2}+2 b-1\right)}{(1+b)^{3}} .
$$

The function (2) maps $U$ onto $U \backslash(-1,0]$, is univalent and symmetric in $U$, $L(0)=b$, and therefore $L \in \mathcal{B}_{0}^{(R)}(b)$. Let further $S_{1}^{(R)}$ denote the family of

1991 Mathematics Subject Classification: Primary 30C45.

Key words and phrases: univalent function, Loewner differential equation. 
all functions $f$ which are analytic, univalent and symmetric in $U$ and satisfy $f(U) \subset U, f(0)=0$. It is obvious that if $f \in S_{1}^{(R)}$ then $L \circ f \in \mathcal{B}_{0}^{(R)}(b)$. But also conversely, if $F \in \mathcal{B}_{0}^{(R)}(b)$ then $F(U) \cap(-1,0]=\emptyset$, hence $L^{-1} \circ F \in S_{1}^{(R)}$. Moreover, any $F \in \mathcal{B}_{0}^{(R)}(b)$ is subordinate to $L$. The above relations allow the application of Loewner's theory, adapted to the class $S_{1}^{(R)}$ by O. Tammi [7], pp. $61-77$, to functions of the class $\mathcal{B}_{0}^{(R)}(b)$. It turns out that in this manner it is possible not only to obtain estimates of $A_{2}$ in the class $\mathcal{B}_{0}^{(R)}(b)$ in an easier way than using the variational method as in [6], but also to obtain all the extremal functions.

2. Loewner's theory applied to $\mathcal{B}_{0}^{(R)}(b)$. $D$ is called a symmetric 2 -slit disk if it is obtained from the disk $U$ by removing two Jordan arcs not containing 0 , symmetric about the real axis and such that $D$ is a simply connected domain. It is known that each simply connected domain, included in the disk $U$, symmetric about the real axis and containing 0 , can be approximated, in the sense of convergence towards a kernel, by domains like ones considered above, and hence on account of the Carathéodory Convergence Theorem, each function in $S_{1}^{(R)}$ can be approximated in the topology of uniform convergence on compact sets by $S_{1}^{(R)}$ functions that map $U$ onto symmetric 2 -slit disks. Hence the set of all such functions - denote it by $\mathcal{S}$ is dense in $S_{1}^{(R)}$ and the infimum and supremum in $S_{1}^{(R)}$ of any functional (real and continuous) are the same in $S_{1}^{(R)}$ as in $\mathcal{S}$.

Tammi [7], p. 68, proved the following theorem for functions of class $\mathcal{S}$.

TheOREM I. For each symmetric 2-slit domain $D$ there exists a function $\vartheta=\vartheta(u)$, continuous in $\left[u_{0}, 1\right], u_{0}>0$, which determines a differential equation

$$
u \frac{\partial f(z, u)}{\partial u}=\frac{f(z, u)-f^{3}(z, u)}{1-2 \cos \vartheta(u) f(z, u)+f^{2}(z, u)},
$$

so that its solution $f\left(z, u_{0}\right)$ with the initial condition $f(z, 1)=z$ is the mapping function of $U$ onto $D$ with $f\left(0, u_{0}\right)=0$.

Conversely, if $\vartheta$ is continuous in $\left[u_{0}, 1\right]$ for some $u_{0}>0$ and (3) is integrated with the initial condition $f(z, 1)=z$, then the solution satisfies $f(z, u) \in S_{1}^{(R)}, f_{z}^{\prime}(0, u)=u$.

Denoting by $\mathcal{S}_{1}$ the set of all solutions of the equations (3) with the functions $\vartheta$ continuous in $\left[u_{0}, 1\right]$ for some $u_{0}>0$ and with the initial condition $f(z, 1)=z$, we have $\mathcal{S} \subset \mathcal{S}_{1} \subset S_{1}^{(R)}$. The continuity of the function $L$ implies that the family $\mathcal{L}=\left\{F: F=L \circ f\right.$ for some $\left.f \in \mathcal{S}_{1}\right\}$ is dense in the class $\mathcal{B}_{0}^{(R)}(b)$, and hence if $\mathcal{F}$ is a functional real, continuous and bounded 
in $\mathcal{B}_{0}^{(R)}(b)$, then

$$
\inf _{\mathcal{B}_{0}^{(R)}(b)} \mathcal{F}=\inf _{\mathcal{L}} \mathcal{F}, \quad \sup _{\mathcal{B}_{0}^{(R)}(b)} \mathcal{F}=\sup _{\mathcal{L}} \mathcal{F}
$$

3. Lower and upper bounds of $A_{2}$. Let

$$
f(z, u)=u\left(z+a_{2}(u) z^{2}+a_{3}(u) z^{3}+\ldots\right)
$$

satisfy the equation (3) and the initial condition $f(z, 1)=z$ with some $\vartheta$ continuous in $\left[u_{0}, 1\right]$ for some $u_{0}>0$. Let

$$
F(z, u)=L(f(z, u))=b+A_{1}(u) z+A_{2}(u) z^{2}+\ldots
$$

By $(3), a_{2}^{\prime}(u)=2 \cos \vartheta(u)$, and hence

$$
a_{2}(u)=-2 \int_{u}^{1} \cos \vartheta(t) d t, \quad u_{0} \leq u \leq 1 .
$$

From (4), (2) and $\left(2^{\prime}\right)$ it follows that

$$
\begin{aligned}
A_{1}(u) & =B_{1} u=\frac{4 b(1-b)}{1+b} u, \\
A_{2}(u) & =B_{1} u a_{2}(u)+B_{2} u^{2} \\
& =\frac{-8 b(1-b)}{1+b}\left(u \int_{u}^{1} \cos \vartheta(t) d t+\frac{b^{2}+2 b-1}{(1+b)^{2}} u^{2}\right) .
\end{aligned}
$$

It is obvious that $A_{2}(u)$ is maximal if $\cos \vartheta(t)=-1$ and it is minimal if $\cos \vartheta(t)=1$ for $u \leq t \leq 1$. Thus we obtain the following inequality:

$$
\text { (5) } \begin{aligned}
\frac{-8 b(1-b)}{(1+b)} & \left(u-\frac{2}{(1+b)^{2}} u^{2}\right) \\
& \leq A_{2}(u) \leq \frac{-8 b(1-b)}{(1+b)}\left(u-\frac{2 b^{2}+4 b}{(1+b)^{2}} u^{2}\right), \quad 0 \leq u \leq 1 .
\end{aligned}
$$

Both inequalities are sharp. The right-hand side of (5) attains its maximal value for $u^{*}=(1+b)^{2} /(4 b(b+2))$. If $u^{*} \leq 1$, that is, if $2 / \sqrt{3}-1 \leq b<1$, then

$$
\max _{u \in[0,1]} A_{2}(u)=A_{2}\left(u^{*}\right)=\frac{1-b^{2}}{b+2} .
$$

If $u^{*}>1$, that is, if $0<b \leq 2 / \sqrt{3}-1$, then

$$
\max _{u \in[0,1]} A_{2}(u)=A_{2}(1)=-\frac{8 b(1-b)}{(1+b)^{3}}\left(b^{2}+2 b-1\right)=B_{2} .
$$

The left-hand side of (5) attains its minimal value for $u^{* *}=(1+b)^{2} / 4 \leq 1$, 
hence for every $0<b<1$,

$$
\min _{u \in[0,1]} A_{2}(u)=A_{2}\left(u^{* *}\right)=-b\left(1-b^{2}\right) .
$$

Exactly the same results were obtained in $[6]$ by means of the variational method.

Let us now find functions whose second coefficient satisfies the equalities in (6), (7) and (8).

Putting in $(3) \cos \vartheta(u)=-1$ for $u \in\left[u_{0}, 1\right], u_{0}>0$ arbitrary, we get the identity

$$
\frac{f(z, u)+1}{f(z, u)(1-f(z, u))} \frac{\partial f(z, u)}{\partial u}=\frac{1}{u},
$$

where $f$ is the function from the second part of Theorem I.

Integrating (9) from $u_{1}$ to 1 , where $u_{1}=u^{*}$ for $0<b \leq-1+\frac{2}{3} \sqrt{2}$ and $u_{1}=1$ for $2 / \sqrt{3}-1<b<1$, we obtain

$$
\frac{f\left(z, u_{1}\right)}{(1-f(z, u))^{2}}=u_{1} \frac{z}{(1-z)^{2}} .
$$

If $u_{1}=1$ then $f\left(z, u_{1}\right)=f(z, 1)=z$ and $F(z)=L(z)$, hence for $0<b \leq$ $2 / \sqrt{3}-1$ the function (2) maximizes the second coefficient $A_{2}$. If $2 / \sqrt{3}-1<$ $b<1$ then $A_{2}$ is maximal, by (10), for the function

$$
F(z)=L\left(f\left(z, u^{*}\right)\right)=K^{-1}\left(\frac{4 b}{(1-b)^{2}}\left(\frac{(1+b)^{2}}{4 b(b+2)} \frac{z}{(1-z)^{2}}+\frac{1}{4}\right)\right),
$$

which maps the disk $U$ on $U \backslash(-1, c]$, where

$$
c=\frac{\left(2 b^{3}+3 b^{2}+3\right) \sqrt{2+b}-2(2+b)\left(1-b^{2}\right) \sqrt{1+b}}{\sqrt{2+b}\left(3 b^{2}+6 b-1\right)} .
$$

We see that $c$ tends to 0 as $b \rightarrow(2 / \sqrt{3}-1)-0$.

Putting now in $(3) \cos \vartheta(u)=1$ for $u \in\left[u_{0}, 1\right], u_{0}>0$ arbitrary, we get for the function $f$ satisfying (3) the identity

$$
\frac{1-f(z, u)}{f(z, u)(1+f(z, u))} \frac{\partial f(z, u)}{\partial u}=\frac{1}{u} .
$$

Integrating (12) from $u^{* *}$ to 1 we obtain

$$
\frac{f\left(z, u^{* *}\right)}{\left(1+f\left(z, u^{* *}\right)\right)^{2}}=u^{* *} \frac{z}{(1+z)^{2}} .
$$

The coefficient $A_{2}$ is minimized by the function

$$
F(z)=L\left(f\left(z, u^{* *}\right)\right)=K^{-1}\left(\frac{b}{(1-b)^{2}} \frac{1}{1-(1+b)^{2} z /(1+z)^{2}}\right) .
$$


This function maps the disk $U$ onto $U \backslash((-1,0] \cup[d, 1))$, where

$$
d=\frac{3+6 b^{2}-b^{4}-\left(1-b^{2}\right) \sqrt{9-10 b^{2}+b^{4}}}{8 b} .
$$

We see that $d$ tends to 1 as $b$ tends to 1 .

We now restate the result obtained above:

Theorem. If $F \in \mathcal{B}_{0}^{(R)}(b), 0<b<1$, then

$$
-b\left(1-b^{2}\right) \leq A_{2} \leq \begin{cases}-\frac{8 b(1-b)}{(1+b)^{3}}\left(b^{2}+2 b-1\right) & \text { for } 0<b \leq \frac{2}{\sqrt{3}}-1, \\ \frac{1-b^{2}}{b+2} & \text { for } \frac{2}{\sqrt{3}}-1<b<1 .\end{cases}
$$

The left-hand bound is realized by the function (13) and the right-hand bounds by the functions (11) and (2).

\section{References}

[1] P. Duren and G. Schober, Nonvanishing univalent functions, Math. Z. 170 (1980), 195-216.

[2] C. Horowitz, Coefficients of nonvanishing functions in $H^{\infty}$, Israel J. Math. 30 (1978), 285-291.

[3] J. Hummel, S. Scheinberg and L. Zalcman, A coefficient problem for bounded nonvanishing functions, J. Anal. Math. 31 (1977), 169-190.

[4] J. Krzyż, Coefficient problem for bounded nonvanishing functions, Ann. Polon. Math. 70 (1968), 314.

[5] D. V. Prokhorov and J. Szynal, Coefficient estimates for bounded nonvanishing functions, Bull. Acad. Polon. Sci. Sér. Sci. Math. 29 (1981), 223-230.

[6] J. Śladkowska, On univalent, bounded, non-vanishing and symmetric functions in the unit disk, Ann. Polon. Math. 64 (1996), 291-299.

[7] O. Tammi, Extremum Problems for Bounded Univalent Functions, Lecture Notes in Math. 646, Springer, 1978.

Institute of Mathematics

Silesian Technical University

ul. Kaszubska 23

44-100 Gliwice, Poland

E-mail: matfiz@zeus.pol.sl.gliwice.pl 